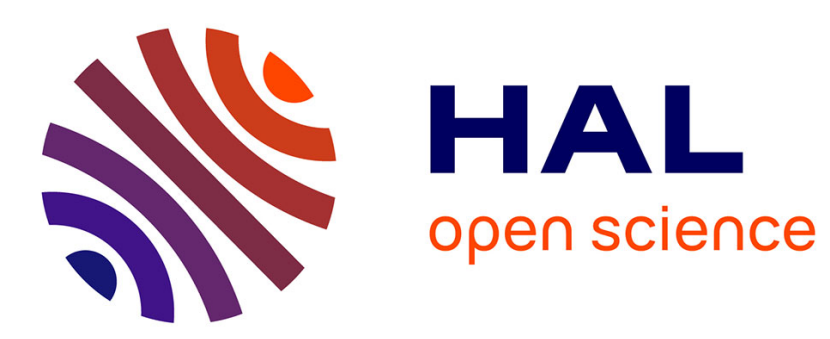

\title{
Numerical study of thermomagnetic cycle
}

Morgan Almanza, Alexandre Pasko, Frédéric Mazaleyrat, Martino Lobue

\section{To cite this version:}

Morgan Almanza, Alexandre Pasko, Frédéric Mazaleyrat, Martino Lobue. Numerical study of thermomagnetic cycle. Journal of Magnetism and Magnetic Materials, 2016, 10.1016/j.jmmm.2016.11.058 . hal-01400798

\section{HAL Id: hal-01400798 \\ https://hal.science/hal-01400798}

Submitted on 29 Nov 2016

HAL is a multi-disciplinary open access archive for the deposit and dissemination of scientific research documents, whether they are published or not. The documents may come from teaching and research institutions in France or abroad, or from public or private research centers.
L'archive ouverte pluridisciplinaire HAL, est destinée au dépôt et à la diffusion de documents scientifiques de niveau recherche, publiés ou non, émanant des établissements d'enseignement et de recherche français ou étrangers, des laboratoires publics ou privés. 


\title{
Numerical study of thermomagnetic cycle
}

\author{
Morgan Almanza ${ }^{1}$, Alexandre Pasko $^{1}$, Frédéric Mazaleyrat ${ }^{1}$, Martino LoBue ${ }^{1}$ \\ ${ }^{1}$ SATIE, ENS Cachan, CNRS, Université Paris-Saclay, 94235 Cachan, France \\ morgan.almanza@ens-cachan.fr
}

\begin{abstract}
We estimate the efficiency and power of a thermal energy harvesting thermodynamic cycle using a magnetocaloric material as active substance. The thermodynamic cycle is computed using an equation of state, either extrapolated from experimental data or deduced using a phenomenological Landau model. The magnetic work is then compared to the maximum work. Afterwards power is estimated using a simple thermal exchange model. Simulations of different cycles for different working points illustrate the tradeoff between power and efficiency.
\end{abstract}

Index Terms-magnetocaloric materials, thermal energy harvesting, thermomagnetic cycle, simulation

\section{INTRODUCTION}

Nowadays much of the industrial energy consumption consists in thermal processes, such as cement and steel production, where about one third of the used energy is discarded as low grade heat. The supply of waste heat represents a huge and freely available amount of energy to make it a key target for energy conversion technologies, notwithstanding the small thermodynamic efficiency to be expected because of the thermodynamic limit. Unfortunately, a thermal energy harvesting system with high power density and/or efficiency does not currently exist. Many efforts have been done on thermoelectric (TE) systems [1]. Energy harvesting systems from waste heat based on thermomagnetic generation (TMG) have been studied since the 1948 paper by Brillouin and Iskenderian [2] and the patents by Tesla (1890) and Edison (1892). The advent of giant magnetocaloric materials (MCM) imposes a fresh assessment of the potential of TMG in terms of efficiency and power and an increasing interest towards this opportunity is apparent from recent publications [2, 3]. As in the case of TE, an issue too often neglected is that a tradeoff exists between efficiency and power density [3]. In this paper we focus on this aspect presenting numerical simulations, based on a simple thermal exchange model, to estimate efficiency and power density.

TMG from waste heat involves harnessing the pyromagnetic effect (PE), namely the induction of a magnetization by the action of a temperature change. Two approaches are commonly envisaged: the first consists in cycling the active material around ferromagnetic-paramagnetic transition while temperature change between the hot and cold reservoirs produces a magnetization change in time which drives an electric current [4]; the second uses the magnetization change in the active substance and the related force/couple exerted on a secondary material to directly produce mechanical work [5]. Efficiency and power density depend on many parameters: the heat exchanger, the material, the magnetic field available, the thermodynamic cycle used and the way we convert the magnetization change to electrical energy. Here we propose to examine in detail the tradeoff between efficiency and power on a single Ericsson cycle working between two heat sources, namely a hot one and a heat sink. Eventually the possibility to use the same approach to regenerative multiple heat sources cycle will be discussed.

\section{MATERIAL MODELING}

MCM are classified following the order of the phase transition driving the effect. Second order materials present a continuous ferromagnetic to paramagnetic transition at the Curie temperature $T_{C}$, whereas the first order ones show an abrupt transition with a discontinuity in the magnetization versus temperature curve. Besides, a strong magneto-elastic coupling and sometimes a structural transition are associated with the first order magnetic phase change. As in most of first order phase transitions, hysteresis and metastability may be present. The most promising MCMs are the La-Fe-Si and the Mn-Fe-P$\mathrm{Si}$ based compounds [6]. These materials show a very sharp transition with critical temperature adjustable with compositions and interstitial modification. Some of these compounds are available at industrial scale.

The estimation of TMG performance is based on the accuracy of the material modeling. Historically, Brillouin used CurieWeiss law for second order materials [2]. Weiss-DebyeSommerfeld model would be better taking into account the effect of field and temperature on thermal capacity. Here, data from a real material, $\operatorname{Pr}_{0.65} \mathrm{Sr}_{0.35} \mathrm{MnO}_{3}$ [7], will be used too. Characterization of this compound published in the literature is very detailed [7], the demagnetizing field in the calorimetric and the magnetometric measurements has been corrected, and therefore specific entropy $s$ obtained with data treatment can be considered as a reliable state function. For first order material we shall use a Landau approach where magnetoelastic coupling is taken into account.

\section{THERMODYNAMIC CYCLE AND SIMULATIONS}

Some thermomagnetic cycles are shown in Fig. 1. The material is assumed to be always at thermodynamic equilibrium (i.e. the phase transition, whatever its order, is assumed to take place at equilibrium). Therefore, assuming the usual entropy balance expression, $d s=d s_{e}+d s_{i}$, where $d s_{e}=\delta Q / T$ represents the reversibly exchanged specific entropy, and $d s_{i} \geq 0$ is the entropy production associated with irreversible heat exchange.

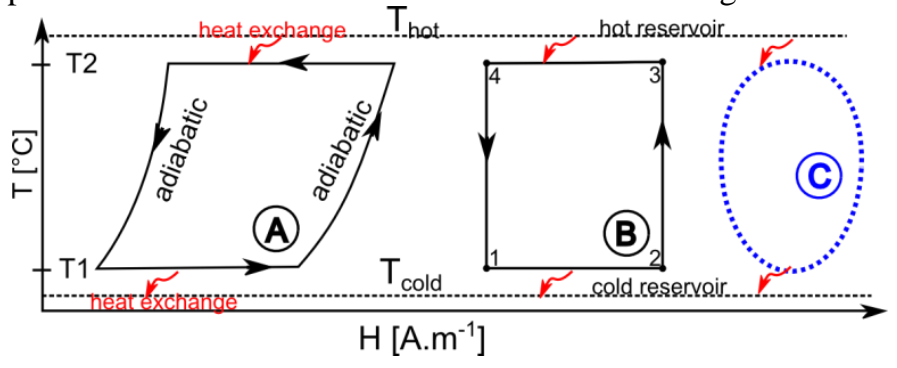


Fig. 1. Examples of two ideal cycles: (A) isotemperature-adiabatic, (B) isotemperature-isofield, and of a generic cycle (C).

From standard thermodynamic relations applied to an elementary volume, we can write,

$$
\begin{gathered}
d u=\delta Q-\vec{M} d \vec{B} \\
T d s=c_{H} d T+T \frac{\partial s}{\partial H} d H=\delta Q
\end{gathered}
$$

where $u$ is the internal energy, $-\vec{M} d \vec{B}$ the magnetic work, $Q$ the heat exchange, $s$ the entropy and $c_{H}$ the heat capacity at constant field, all these quantities being defined per unit volume. The efficiency of the cycles, defined by how much work is produced compared to the heat absorbed, depends only on the $T, H$ path. Here the field $H$ goes from 0 to $1 \mathrm{~T}$,

$$
\eta=-\frac{W_{\text {mag }}}{Q_{\text {hot }}}=1+\frac{Q_{\text {cold }}}{Q_{\text {hot }}}
$$

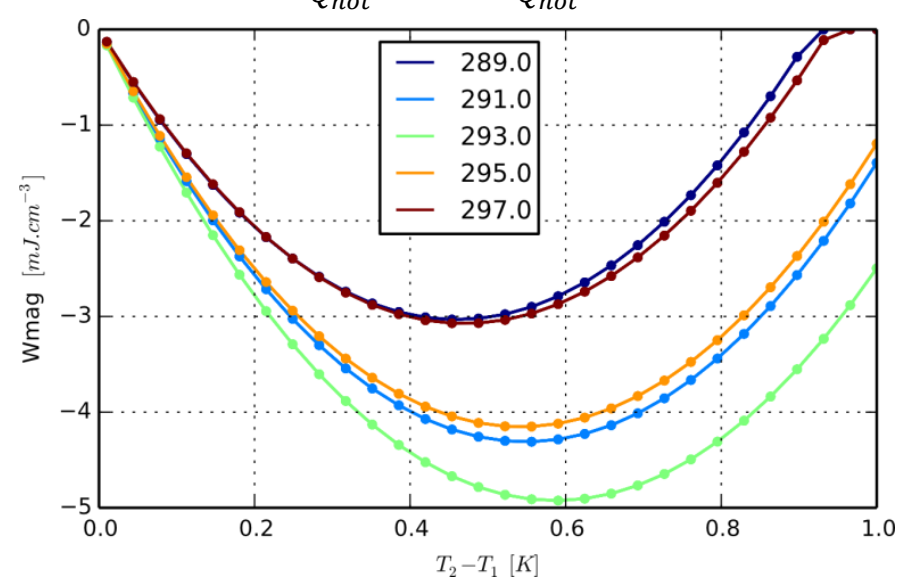

Fig. 2 Magnetic work for the cycle (A) for different $T_{1}$ temperatures indicated in the legend with an applied field of $1 \mathrm{~T}$ using $\mathrm{Pr}_{0.65} \mathrm{Sr}_{0.35} \mathrm{MnO}_{3}$ data [7].

The maximum work that could be different from the maximum power is obtained when the difference between the maximum and minimum temperatures in the cycle, $T_{2}-T_{1}$, is equal to the half of the adiabatic temperature change. However here we are still using quasi-static purely reversible transformations. To estimate the power associated with the thermodynamic cycle, we have to go beyond this approach by introducing a thermal exchange model as well as finite change rate of the relevant quantities. In this way we estimate the degree of departure from thermal equilibrium between the MCM and the heat sources, namely we have to take into account the temperature difference between the material and the reservoirs lead by the field rate and the thermal exchange characteristic time. Trajectories in the $(T, H)$ path are different and irreversible heat exchanges arise contributing to $d s_{i}$.

\section{POWER AND EFFICIENCY}

To estimate the power $P_{\text {mag }}=W_{\text {mag }} / \Delta t_{\text {period }}$, we use a simple heat exchange model to compute the actual period $\Delta t_{\text {period }}$ of the cycle. The following equation describes the thermal exchange with the two reservoirs,

$$
\delta Q=\left[-k_{\text {hot }}\left(T-T_{\text {hot }}\right)-k_{\text {cold }}\left(T-T_{\text {cold }}\right)\right] d t
$$

where $T_{\text {cold }}, T_{\text {hot }}$, and $T$ are the temperatures of the heat sink, the hot reservoir and the material respectively, and $k_{\text {hot }}(T, H)$ and $k_{\text {cold }}(T, H)$ in $\left[W \cdot K^{-1} \cdot m^{-3}\right]$ represent the thermal contact constants with the hot and cold reservoirs respectively. When the MCM is in thermal contact with the hot reservoir, then $k_{\text {hot }}$ is greater $\left(k_{\text {high }}\right)$ than $k_{\text {cold }}$ which is null $\left(k_{\text {low }}\right)$, and vice versa.

hot reservoir
$\mathrm{k}_{\text {hot }}=k_{\text {high }}=1 \mathrm{~W}_{\mathrm{cm}} \mathrm{cm}^{-3} \mathrm{~K}^{-1}$ thermath
MContact with the hot reservoir
$\mathrm{MCM}$ thickness $\mathrm{e}=1 \mathrm{~mm}$
$\mathrm{k}_{\text {cold }}=\mathrm{k}_{\text {low }}=0$ thermal insulation
with the cold reservoir
cold reservoir

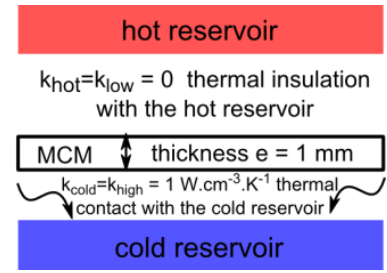

Fig. 3. Different phases of the thermal exchange

The shape of the cycle was imposed in $(T, H)$ form, with the state of $k_{\text {hot }}(T, H)$ and $k_{\text {cold }}(T, H)$, therefore the time variation $d t$ is integrated using the material model (i.e. the material equation of state), and substituting Eq. (2) in Eq. (4),

$$
d t=\frac{c_{H} d T+T \frac{\partial s}{\partial H} d H}{\left[-k_{\text {hot }}\left(T-T_{\text {hot }}\right)-k_{\text {cold }}\left(T-T_{\text {cold }}\right)\right]}
$$

Here we compute the period by imposing the shape of the cycle in the $(H, T)$ plane as represented in Fig. 4.

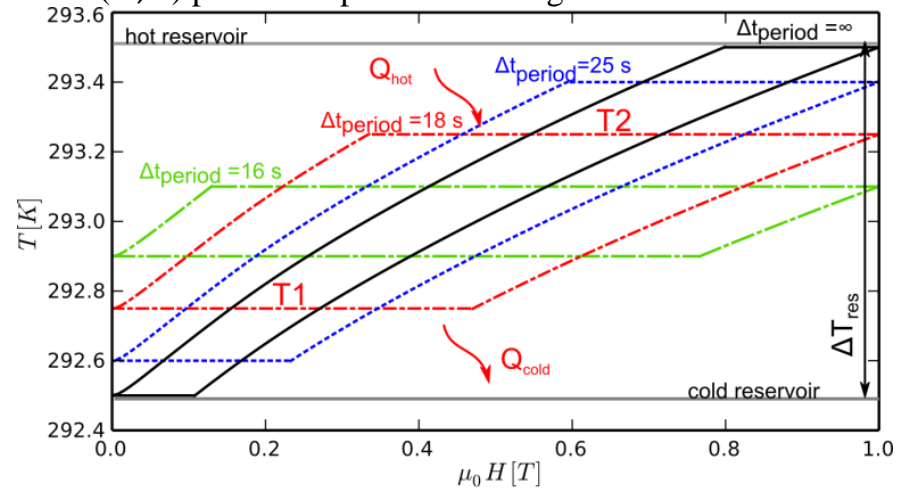

Fig. 4. Example of (A) cycle with its period $\Delta t_{\text {period }}$ where different temperature differences are used for the heat exchange.

The constant $k_{\text {high }}$ is expected to assume values of about $1 \mathrm{~W}$. $\mathrm{cm}^{-3} \cdot \mathrm{K}^{-1}$ using $1 \mathrm{~mm}$ thickness sheet of MCM with $1000 \mathrm{~W} \cdot \mathrm{m}^{-2} \cdot \mathrm{K}^{-1}$ heat transfer coefficient, corresponding to air layer of $25 \mu \mathrm{m}$ shaped by a mechanical contact and the surface roughness. Moreover, the thermal conductivity of the MCM is sufficiently high, i.e higher than $0.5 W \cdot K^{-1} \cdot m^{-1}$, to neglected the heat resistance of MCM. The constant $k_{\text {low }}$ depends on the thermal leakage, i.e. the heat insulation; here we assume it to be vanishing but it should be adjusted according to the system studied.

To evaluate the degree of departures from thermal equilibrium, the maximum work cycle, the entropy production due to heat exchange has to be considered,

$$
\delta s_{i}(T, H)=\left(\frac{1}{T}-\frac{1}{T_{r e s}(T, H)}\right) \delta Q(T, H)
$$

The system studied is now the MCM with its sources. The temperature of the source $T_{\text {res }}$ with which the MCM exchanges is alternatively $T_{\text {hot }}$ and $T_{\text {cold }}$. For example, in (A) cycle, the entropy production is due to irreversible heat exchange along 
the transformation at constant temperature. The entropy follows:

$$
\eta=1-\frac{T_{\text {cold }}}{T_{\text {hot }}}-\frac{s_{i} T_{\text {cold }}}{Q_{\text {hot }}}=\eta_{\text {Carnot }}-\frac{s_{i} T_{\text {cold }}}{Q_{\text {hot }}}
$$

The relative efficiency $\eta_{\text {rel }}$ is the ratio of the cycle efficiency to the efficiency of the Carnot cycle:

$$
\eta_{\text {rel }}=\frac{\eta}{\eta_{\text {Carnot }}}=1-\frac{s_{i} T_{\text {cold }}}{\eta_{\text {Carnot }} Q_{\text {hot }}}
$$

Using thermodynamic relations and the equation of state of the material, we compute the power density and the efficiency using four different temperatures, the ones of the reservoirs $T_{\text {hot }}$ and $T_{\text {cold }}$ and the isotemperatures $T_{2}$ and $T_{1}$, as illustrated in Fig. 1. Indeed, we use the term isotemperature to emphasize the difference with respect to usual isothermal reversible transformations.

\section{STUdy OF THE CYCLE (A) WITH PR $\mathrm{PR}_{0.65} \mathrm{SR}_{0.35} \mathrm{MNO}_{3}$}

The cycle (A) is decomposed in four steps: two adiabatic and two heat exchanges at constant temperature during which the field is adjusted in order to maintain the temperature of the MCM, as shown in the following figures.
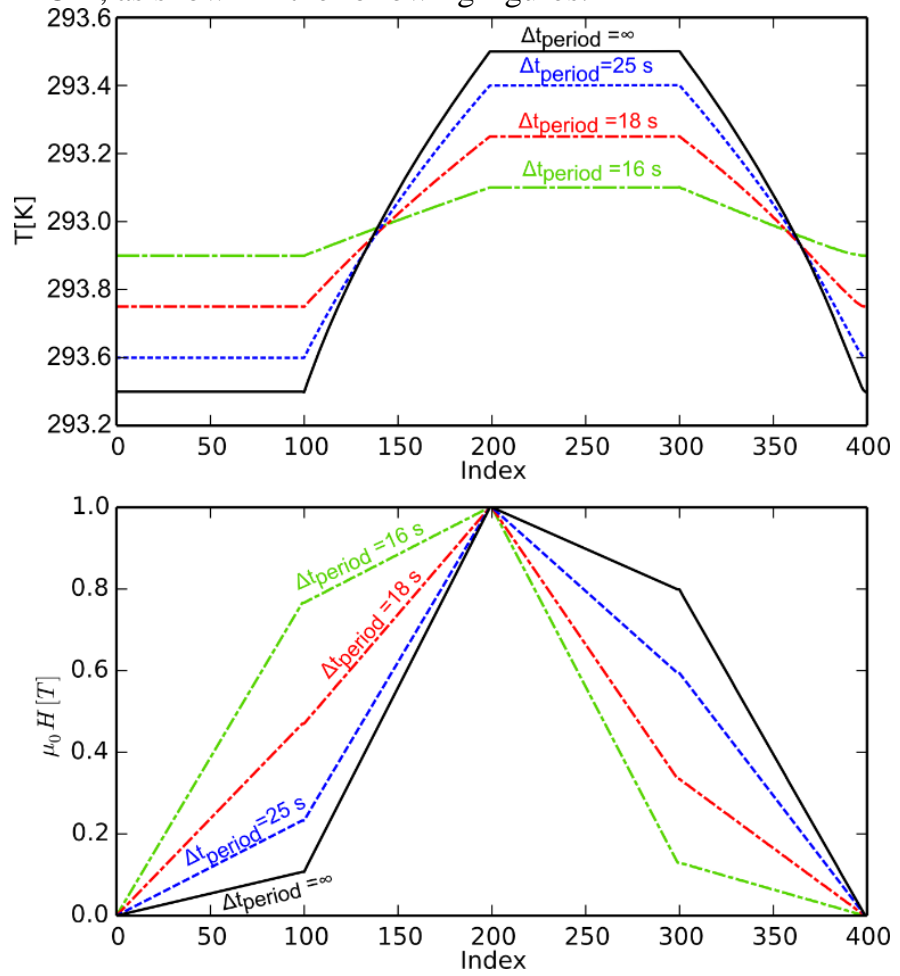

Fig. 5. Field and temperature in the (A) cycle with 100 points in each branch of the cycle, the last index 400 corresponds to $\Delta t_{\text {period }}$.

The relative efficiency decreases with $\eta_{\text {rel }}=1,0.8,0.5,0.2$, from the red cycle to the black one (see Fig. 4) as the period decreases, $\Delta t_{\text {period }}=\infty, 25,18,16 \mathrm{~s}$, and therefore the power produced increases and decreases with respect to $P=$ $0,0.17,0.27,0.17\left[\mathrm{~mW} \cdot \mathrm{cm}^{-3}\right]$, respectively. This simulation on (A) cycle reveals the tradeoff between power and efficiency with an optimum of power when $T_{2}-T_{1}=\Delta T_{\text {res }} / 2$, associated to the results of Fig. 4, with $\Delta T_{\text {res }}=T_{\text {hot }}-T_{\text {cold }}$. In the (A) cycle assuming temperatures greater than the temperature difference $T>\Delta T_{\text {res }}$, and from Eq. (8) and (6), the relative efficiency is:

$$
\eta_{\text {rel }}=\frac{T_{2}-T_{1}}{\Delta T_{\text {res }}}
$$

in agreement with the simulation. It is also equal to the ratio of two Carnot efficiencies, one with isotherms $T_{1}$ and $T_{2}$ and the other with isotherms $T_{\text {cold }}$ and $T_{\text {hot }}$. In (A) cycle the heat exchange with the hot and cold reservoirs is done using the same constant temperature difference. Therefore, the period of the exchange is approximated with,

$$
\Delta t_{\text {period }} \sim \frac{2 Q_{\text {hot }}}{k_{\text {high }}\left(\Delta T_{\text {res }}-\left(T_{2}-T_{1}\right)\right) / 2}
$$

Equation (3) divided by the period $\Delta t_{\text {period }}$ combined with Eq. (9) and (7) gives the power as a function of the relative efficiency,

$$
\begin{gathered}
P\left(\eta_{\text {rel }}\right)=\frac{\Delta T_{\text {res }}^{2} k_{\text {high }}}{4 T_{\text {hot }}} \eta_{\text {rel }}\left(1-\eta_{\text {rel }}\right) \\
P_{\text {max }}=\frac{\left(T_{2}-T_{1}\right)^{2} k_{\text {high }}}{4 T_{\text {hot }}}
\end{gathered}
$$

and therefore explains the trade-off between efficiency and power density. The maximum power is for $\eta_{r e l}=0.5$, i.e. $\Delta T_{\text {res }}=2\left(T_{2}-T_{1}\right)$, for a given $T_{2}-T_{1}$ the user is able to optimize thermal exchange with the sources.

The maximum power density, Eq. (11), depends on $\Delta T_{\text {res }}^{2}=$ $4\left(T_{2}-T_{1}\right)^{2}$, but $T_{2}-T_{1}$ is limited by the magnetic field available, i.e. by the adiabatic temperature change. When $T_{2}-$ $T_{1}$ equals $\Delta T_{\text {adia }}$, the period goes to zero. If $W_{\text {mag }}$ is small then the heat exchange is small and the period decreases, leading to an increase of power, as shown in Eq. (11) and in Fig. 6. In order to take into account actual constraints as the time needed to change the thermal contact between the hot and cold reservoirs, a time is added on the period. Two seconds seems a reasonable technological guess for a contact switch. Fig. 6 shows the maximum work cycle for $T_{1}=293 \mathrm{~K}$ and $T_{2}=$ $293.9 \mathrm{~K}$ which are across the temperature of the transition.

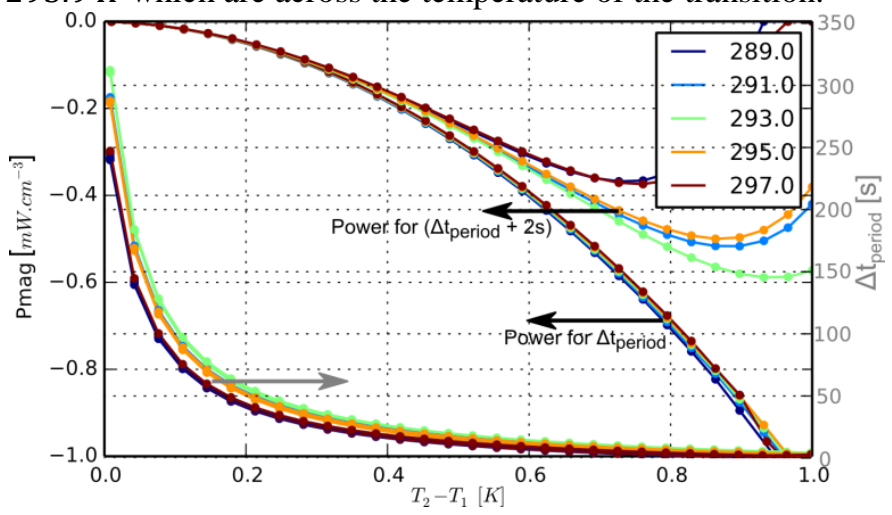

Fig. 6. Magnetic power based on $P_{\text {mag }}=W_{\text {mag }} / \Delta t_{\text {period }}$ and $P_{\text {mag }}=$ $W_{\text {mag }} /\left(\Delta t_{\text {period }}+2[s]\right)$ for (A) cycle (left axis) and $\Delta t_{\text {period }}$ (right axis) for different $T_{1}$ temperatures given in the legend and with the condition $\Delta T_{\text {res }}=$ $2\left(T_{2}-T_{1}\right)$, given $\eta_{\text {rel }}=0.5$.

To circumvent the limited value of $2\left(T_{2}-T_{1}\right)=T_{\text {hot }}-T_{\text {cold }}$, an intermediate reservoir at $\left(T_{\text {hot }}+T_{\text {cold }}\right) / 2$ can be introduced through a cascade of cycles. Another method is to use $\left(T_{2}-T_{1}\right) \ll T_{\text {hot }}-T_{\text {cold }}$, then relative efficiency decreases, 
Eq. (9), and the power density increases, Eq. (11). A cascade has a constant relative efficiency and power density. Even if it lead to more finite heat transfer and thus extra entropy production, the ratio entropy production over $\eta_{\text {Carnot }}$, used in the relative efficiency in Eq. (9), is constant.

\section{StUdy OF THE CYCLE (B) WITH $\mathrm{PR}_{0.65} \mathrm{SR}_{0.35} \mathrm{MNO}_{3}$}

Efficiency and power of cycle (B) with two isotemperatures and two isofields depend on the shape of the cycle, i.e. temperatures $T_{2}$ and $T_{1}$ and those of the reservoirs $T_{\text {hot }}$ and $T_{\text {cold }}$. Magnetic power increases when $\Delta T_{\text {res }}$ increases as shown Fig. 7, when curves are parabolic. That is due to the fact that when $T_{2}-T_{1}=$ $\Delta T_{\text {res }}$ then $T_{\text {period }}$ is infinite, and when $T_{2}-T_{1}=0$, the magnetic energy $W_{\text {mag }}$ produced by the cycle is null.

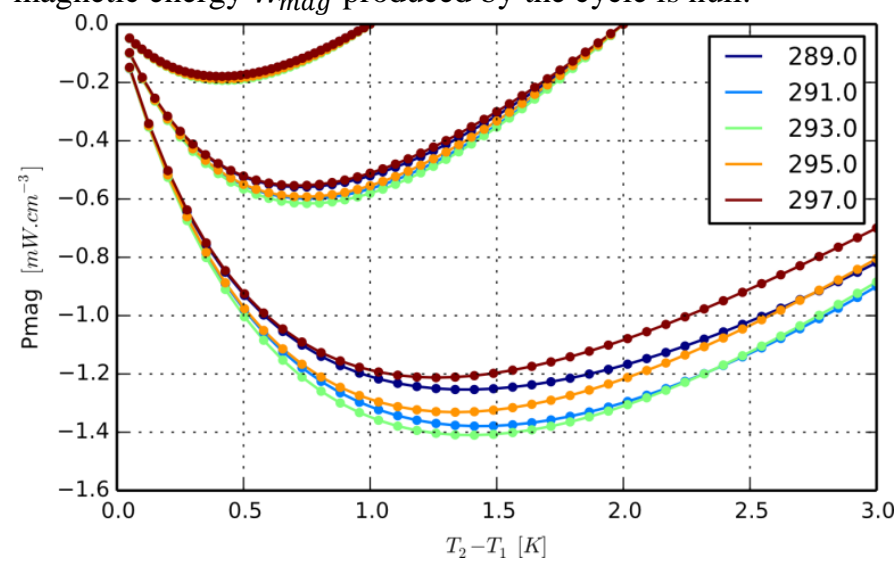

Fig. 7. Magnetic power for different temperatures $T_{1}$ given in the legend and with $\Delta T_{\text {res }}=1,2$ and $4 \mathrm{~K}$ respectively from the top curves to the bottom ones.

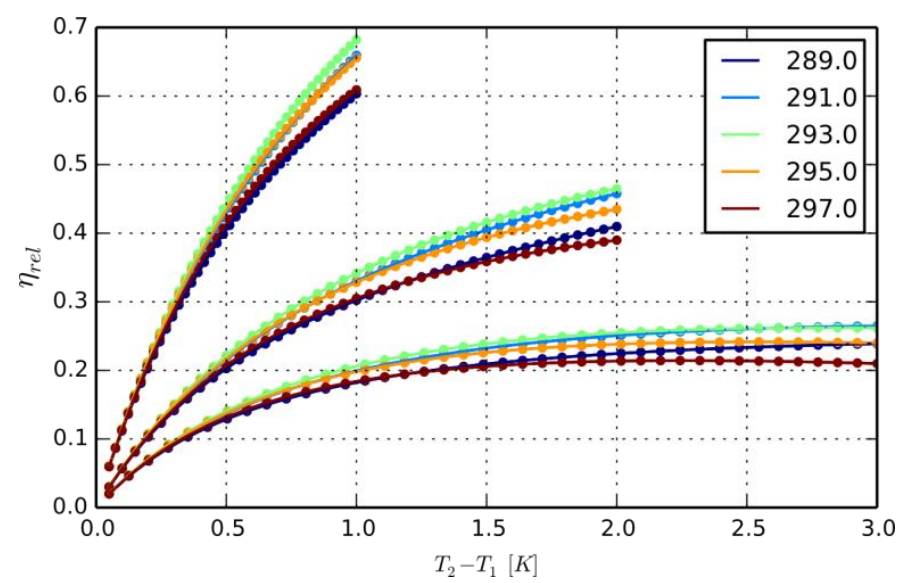

Fig. 8. Relative efficiency for different temperatures $T_{1}$ given in the legend for $\Delta T_{\text {res }}$ from $1 \mathrm{~K}$ for the top curves to $4 \mathrm{~K}$ for the bottom curves.

In Fig. 7 and 8, the $\Delta T_{\text {res }}$ has discrete values 1,2 and $4 K$. Fig. 9 shows the relative efficiency and the magnetic power density as functions of $\Delta T_{\text {res }}$, computed for the optimal cycle, i.e. $T_{2}-$ $T_{1}$, in terms of power density.

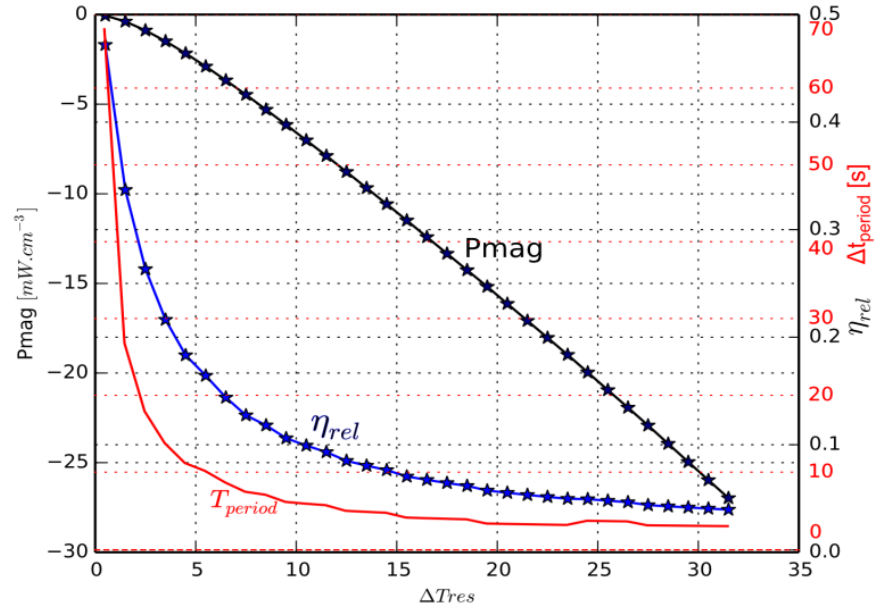

Fig. 9 Maximum power for different $\Delta T_{\text {res }}$ and relative efficiency.

For the comparison of cycles (A) and (B) for a given $\Delta T_{\text {res }}$ we choose the cycle with the optimal power density. In that configuration, the cycle (B) exhibits a tradeoff between efficiency and power different from the one of cycle (A). Indeed, in Fig. 6 the power increases when $\Delta T_{\text {res }}$ becomes closer to $\Delta T_{\text {adia }}$, while the relative efficiency is constant at 0.5 . The main limitation is due to the frequency of cycling that reaches levels difficult to be experimentally achieved. The cycle (B) exhibits a limited increase of the frequency because the heat exchange per cycle does not go to zero, indeed we always have to exchange the heat due to the thermal capacity.

The cycle (B) works for all reservoir temperatures and therefore reaches higher power density, unlike the cycle (A) which has an upper bound defined by $\Delta T_{\text {adia }}$. A cascade of cycles (A) will have the same performance, i.e. same power density and relative efficiency; that is not the case of cycle $B$, as shown in the Fig. 8.

\section{FIRST ORDER MATERIALS}

The first order MCM are the more promising system for TMG; however, because of the hysteresis and the kinetics of the transition, the modeling may present additional difficulties. Of course hysteresis will potentially reduce the efficiency. However hysteresis has been drastically reduced in many alloys using composition and annealing [8], so here we prefer to focus our attention on an ideal material where hysteresis has been mastered. We try to estimate the potential benefits of first order materials using an equilibrium Landau model where the transition is driven by magneto-elastic coupling between two order parameters (magnetic and elastic) as described in $[9,10]$. The parameters of the model are adjusted using a set of measurements on $\mathrm{Mn}_{1.3} \mathrm{Fe}_{0.65} \mathrm{P}_{0.5} \mathrm{Si}_{0.5}$ [10]. Because the model does not fit the thermal capacity at high temperature, a term $C \ln (T)$ is added to the entropy to introduce a constant $C$ in the thermal capacity. Here we use $C$ values similar to the one used in the case of second order MCM (i.e. $3.9 \mathrm{~J} \cdot \mathrm{K}^{-1} \cdot \mathrm{cm}^{-3}$ ). Actually the logarithmic term represents the lattice contribution to entropy. 

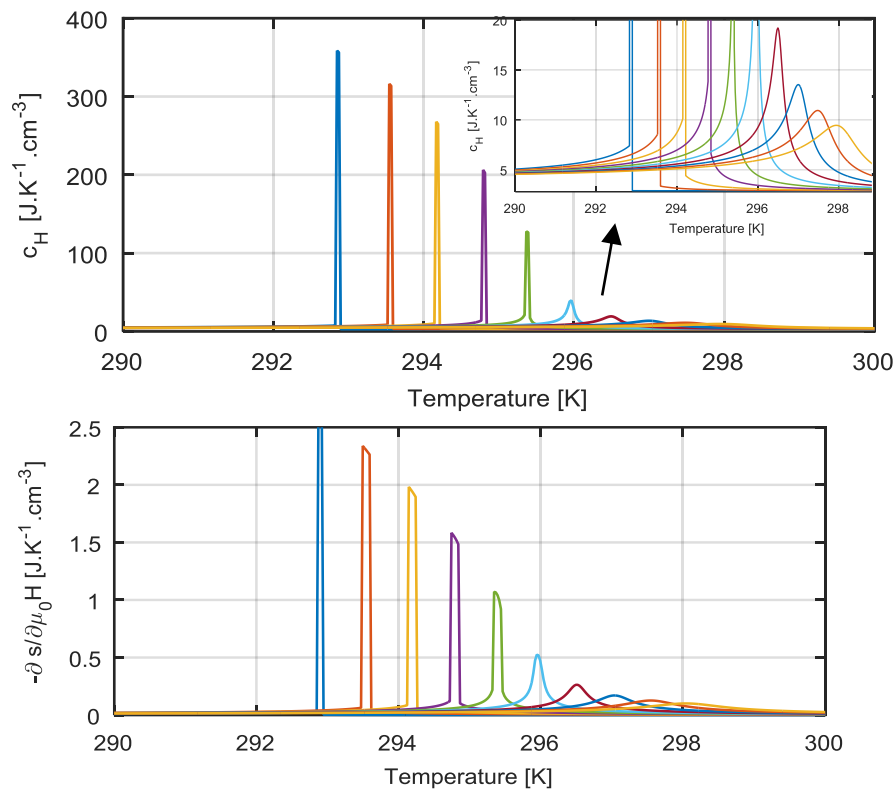

Fig. 10. Characteristics of MCM from the extended Landau model for first order transition.

The thermodynamic cycles (A) and (B) are numerically computed introducing the phase fractions during the transition: the ferromagnetic fraction $x$ and the paramagnetic fraction one $1-x$. As in the previous case, there is a maximum power density when $\Delta T_{\text {res }}=2\left(T_{2}-T_{1}\right)$ with relative efficiency of 0.5 and a maximum of magnetic energy exchanged per cycle at $\Delta T_{\text {adia }} / 2$, as illustrated in Fig. 11 and Fig. 12.

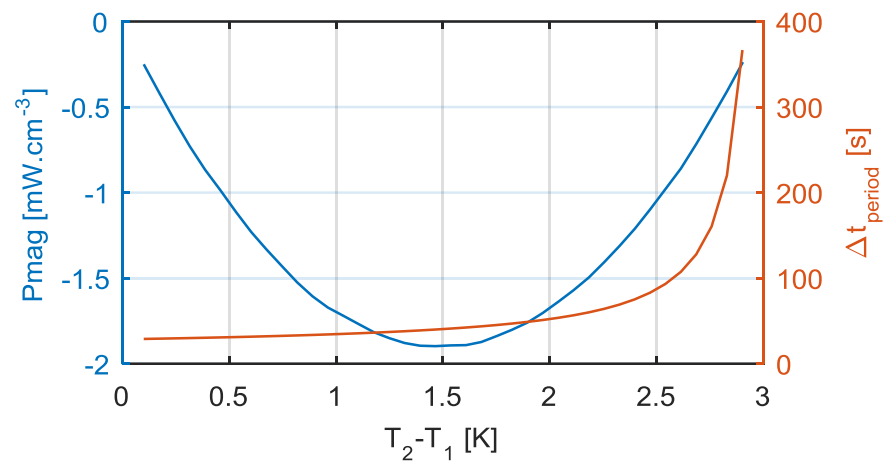

Fig. 11. Power density and time period for the cycle (A), first order MCM, $T_{\text {hot }}=296 \mathrm{~K}$ and $T_{\text {cold }}=293 \mathrm{~K}$ and cycles centered at $294.5 \mathrm{~K}$.

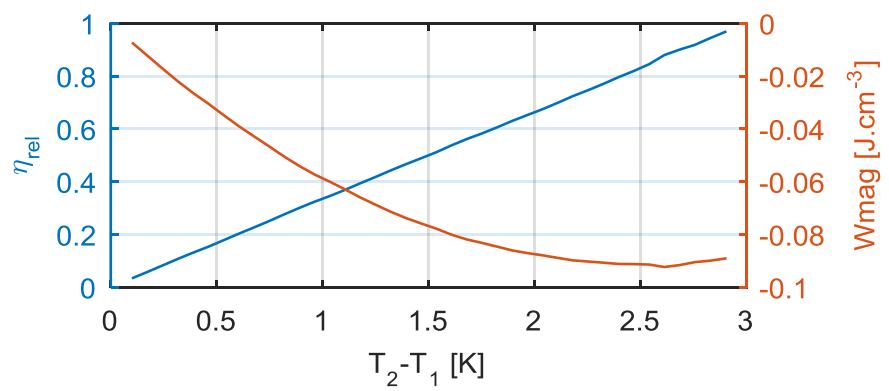

Fig. 12. Efficiency and magnetic energy density under the same conditions as in Fig. 11.

When $T_{2}-T_{1}$ increases, the magnetic energy exchanged per cycle increases because the area enclosed by the cycle in Fig. 13 increases.

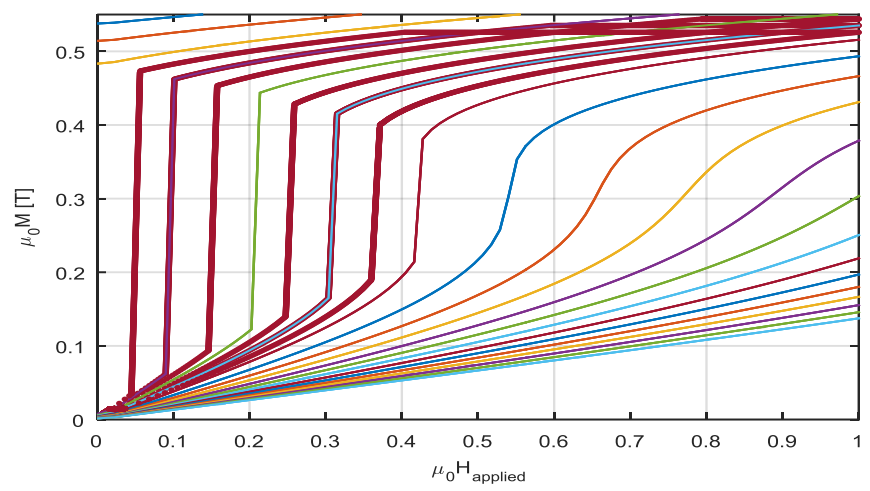

Fig. 13. Example of cycle (A) on magnetization curves at isotemperature with the Landau model for first order MCM.

In Fig. 14 which has to be compared with Fig. 6 for second order MCM, the temperature of the reservoir changes to keep the optimal power point, i.e. $\Delta T_{\text {res }}=2\left(T_{2}-T_{1}\right)$, the maximum power density is similar to that obtained with second order MCM. However $\Delta t_{\text {period }}$ is longer, because the heat exchange is 50 times higher. Therefore a smaller part of the period is required to change the thermal contact, limiting the effect of the $2 \mathrm{~s}$ added in Fig. 6. Eventually the power remains of the same order of magnitude because it is intrinsically limited by the heat exchange coefficient. Keeping the same efficiency, the only way to increase the power density is increasing the heat transfer coefficient to reduce the characteristic heat exchange time. However the increase of power density can be done by increasing $\Delta T_{\text {res }}$, paying a price in terms of entropy production and therefore decreasing the efficiency.

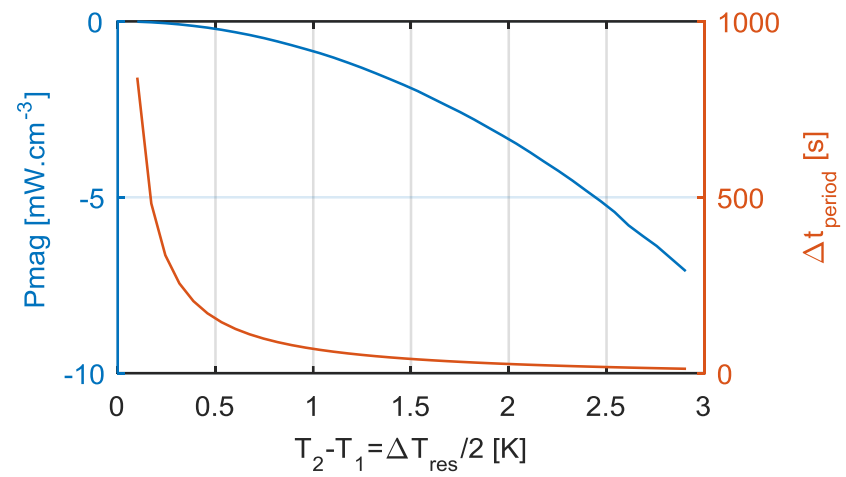

Fig. 14. Magnetic power density and $\Delta t_{\text {period }}$ for different $\Delta T_{\text {res }}=2\left(T_{2}-T_{1}\right)$, with $\eta_{\text {rel }}=0.5$.

With the cycle (B) the efficiency decreases at a slower rate for first order MCM (that is apparent comparing Fig. 15 with Fig. $9)$. This is due to the more intense heat transfer during the isotemperature transformation, related to $\partial s / \partial H$, with respect to the one during the isofield, related to $\partial s / \partial T$. In comparison with the cycle (A), there is a supplement of entropy produced by the heat exchange during the isofield, related to $\partial s / \partial T$, which, divided by $Q_{h o t}$, related to $\partial s / \partial H$, decreases the efficiency following Eq. (8). Moreover, the lattice entropy, added through the constant $C$, decreases the efficiency because it is only dependent on $T$. For the same reason, power density increases, but it is still limited by the heat exchange coefficient. 


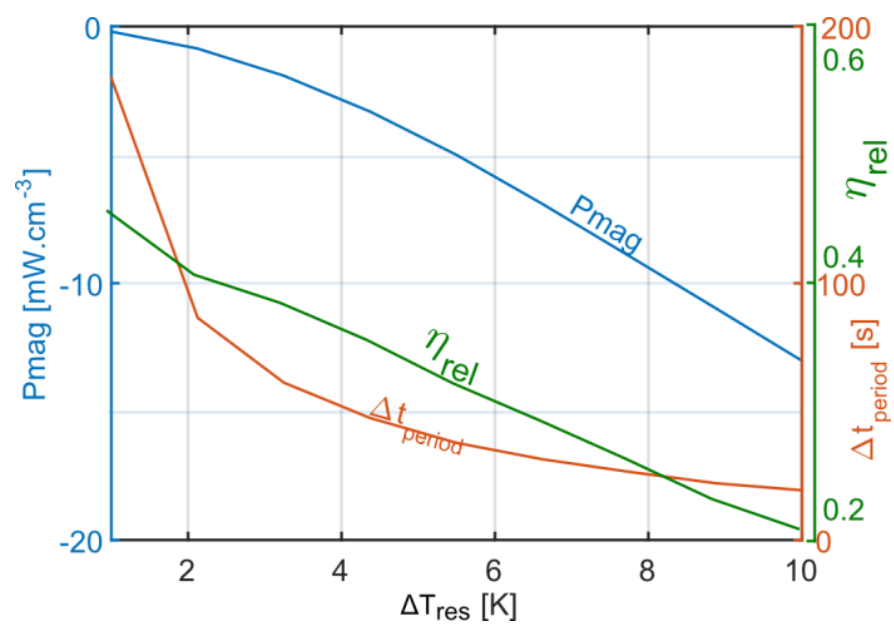

Fig. 15. Maximum power, relative efficiency and the period as functions of the temperature of the reservoir for cycle (B) and first order MCM.

\section{CONCLUSION}

In this paper, comparison of the thermal energy harvesting thermodynamic cycles with estimation of power and efficiency based on a simple heat exchange model has been done using the experimental data for second order material and phenomenological model of the first order material. Power density and efficiency are strongly dependent on the heat exchange coefficient, the temperature of the reservoir and the working point and therefore they should be defined when results are claimed. Power density can be improved by a better heat transfer, and by an increase of the temperature of the reservoir, which inevitably decreases efficiency, highlighting the tradeoff. With the isotemperature-isofield cycle, first order MCM have twice better efficiency and power density than second order MCM. Whereas with the isotemperatureadiabactic cycle, first and second order MCM have similar performances, although the first order transition results in lower working frequency, allowing the conception of a device easier. Further works will be devoted to a more accurate modeling of the first order material, both using experimental data and taking into account the effect of hysteresis. Using the results obtained, comparisons with TEG in [11] show much higher efficiency at maximum power for TMG. In these paper, the impact of the exchanger with the ambient has been ignored. Even if further works are needed, the impact of the exchanger should be significantly less in TMG considering the higher efficiency of TMG.

\section{REFERENCES}

[1] M. T. Dunham, M. T. Barako, S. LeBlanc, M. Asheghi, B. Chen, and K. E. Goodson, "Power density optimization for micro thermoelectric generators," Energy, vol. 93, pp. 2006-2017, Dec. 2015.

[2] L. Brillouin and H. P. Iskenderian, "Thermomagnetic generator," Fed. Telecommun. Lab., 1948.

[3] D. Vuarnoz, A. Kitanovski, C. Gonin, Y. Borgeaud, M. Delessert, M. Meinen, and P. W. Egolf, "Quantitative feasibility study of magnetocaloric energy conversion utilizing industrial waste heat," Appl. Energy, vol. 100, pp. 229-237, Dec. 2012.

[4] T. Christiaanse and E. Brück, "Proof-of-Concept Static Thermomagnetic Generator Experimental Device," Metall. Mater. Trans. E, vol. 1, no. 1, pp. 36-40, Mar. 2014.
[5] C.-J. Hsu, S. M. Sandoval, K. P. Wetzlar, and G. P. Carman, "Thermomagnetic conversion efficiencies for ferromagnetic materials," J. Appl. Phys., vol. 110, no. 12, p. 123923, Dec. 2011.

[6] K. G. Sandeman, "Magnetocaloric materials: The search for new systems," Scr. Mater., vol. 67, no. 6, pp. 566-571, Sep. 2012.

[7] M. Almanza, A. Kedous-Lebouc, J.-P. Yonnet, U. Legait, and J. Roudaut, "Magnetic refrigeration: recent developments and alternative configurations," Eur. Phys. J. Appl. Phys., vol. 71, no. 1, p. 10903, Jul. 2015.

[8] N. T. Trung, Z. Q. Ou, T. J. Gortenmulder, O. Tegus, K. H. J. Buschow, and E. Brück, "Tunable thermal hysteresis in $\mathrm{MnFe}(\mathrm{P}, \mathrm{Ge})$ compounds," Appl. Phys. Lett., vol. 94, no. 10, p. 102513, Mar. 2009.

[9] J.-C. Tolédano and P. Tolédano, The Landau theory of phase transitions: application to structural, incommensurate, magnetic and liquid crystal systems. Singapore: World Scientific, 1987.

[10] A. Pasko, A. Bartok, K. Zehani, L. Bessais, F. Mazaleyrat, and M. LoBue, "X-ray diffraction analysis of the magnetoelastic phase transition in the Mn-Fe-P-Si magnetocaloric alloy," AIP Adv., vol. 6, no. 5, p. 56204, May 2016.

[11] M. Almanza, A. Pasko, A. Bartok, F. Mazaleyrat, and M. LoBue, "Thermal energy harvesting: thermomagnetic versus thermoelectric generator," 7th International Conference on Magnetic Refrigeration at Room Temperature, 13-Sep-2016. 